\title{
Collective flow measurements at RHIC energies
}

\author{
Shinlchi Esumi ${ }^{1}$ a \\ ${ }^{1}$ Division of Physics, Faculty of Pure and Applied Sciences, University of Tsukuba, \\ Center for Integrated Research in Fundamental Science and Engineering (CiRfSE), \\ Tenno-dai 1-1-1, Tsukuba, Ibaraki 305-8571, Japan
}

\begin{abstract}
Recent experimental results on collective flow measurements from relativistic heavy-ion collider (RHIC) are presented and discussed to study high-temperature and high-density quark-nuclear matter, Quark Gluon Plasma (QGP) especially focusing on bulk properties, such as freeze-out parameters, temperature, chemical potential, collective expansion, azimuthal event anisotropy measurements. Their relations to the various correlation and fluctuation studies are also discussed, including initial geometrical and E- and B-field conditions as well as possible collective flow evolution that could even be developed in small systems. Current results and understandings from the beam energy scan program (BES) and future plans are discussed and reviewed.
\end{abstract}

\section{Baryon density, directed flow and initial fields}

Identified particle yields and ratios are used to extract chemical freeze-out temperature $T_{c h}$ and Baryon chemical potential $\mu_{B}$ based on thermal models with chemical freeze-out assumptions, as shown in Fig.1 (left), which confirms that the high temperature system indeed moves towards the high Baryon density region by reducing the colliding beam energy [1]. The thermal kinetic freeze-out parameters $T_{k i n}$ and transverse radial expansion velocity $\beta_{T}$ has been extracted by Blast wave model based on hydrodynamical expansion with a common kinetic freeze-out for all particle species. Fig.1 (right) shows that the system cools down further more by larger radial expansion with increasing the centrality and beam energy [2].

Directed flow gradient vs pseudo-rapidity $\eta$ of anti-proton (top), proton (middle) and net-proton (bottom) are shown in Fig.2 (left) as a function of beam energy, the negative slope of the net-proton $v_{1}$ as a function of $\eta$ might indicate the softening of the equation of state, which could be related to a sudden change of the system pressure close to the critical point [3]. Asymmetric colliding system $\mathrm{Cu}+\mathrm{Au}$ has provided a finite directed flow at mid-rapidity as shown in Fig.2 (right), where the small difference of $v_{1}$ between positive and negative charged particles could be attributed by the initial electric field parallel to the reaction plane inside the participant region expected in the asymmetric collision system [4].

Very strong magnetic fields are expected to be formed in early stage of non-central collisions because of fast moving positive beam ions passing close by. Possible charge asymmetry effects from the strong B-field via 3-particle correlation (like- and un-like signed pair correlation with respect to the

ae-mail: esumi.shinichi.gn@u.tsukuba.ac.jp 

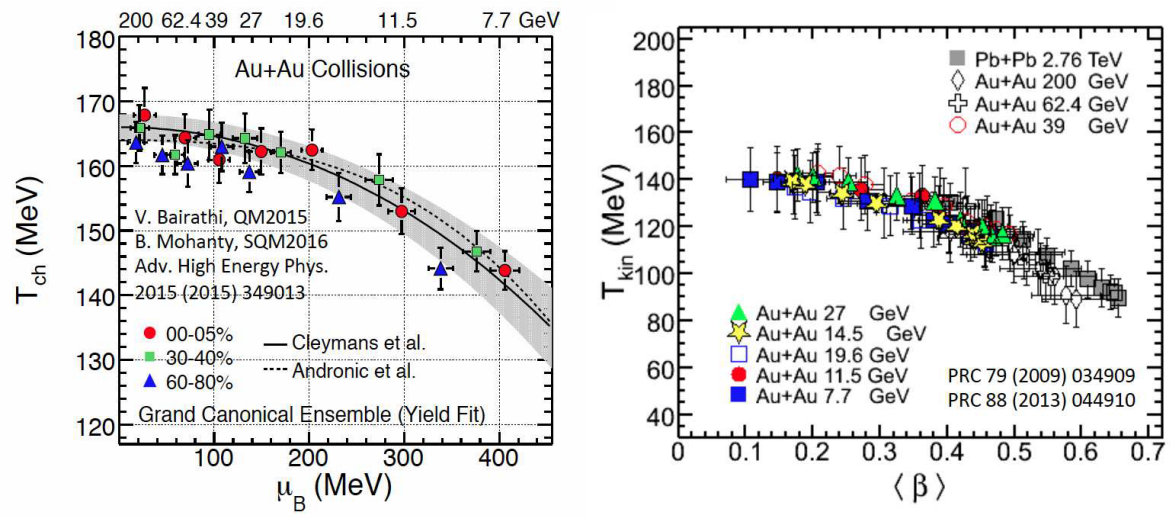

Figure 1. Chemical (left) and kinetic (right) thermal freeze-out parameters as functions of beam energy and centrality $[1,2]$.
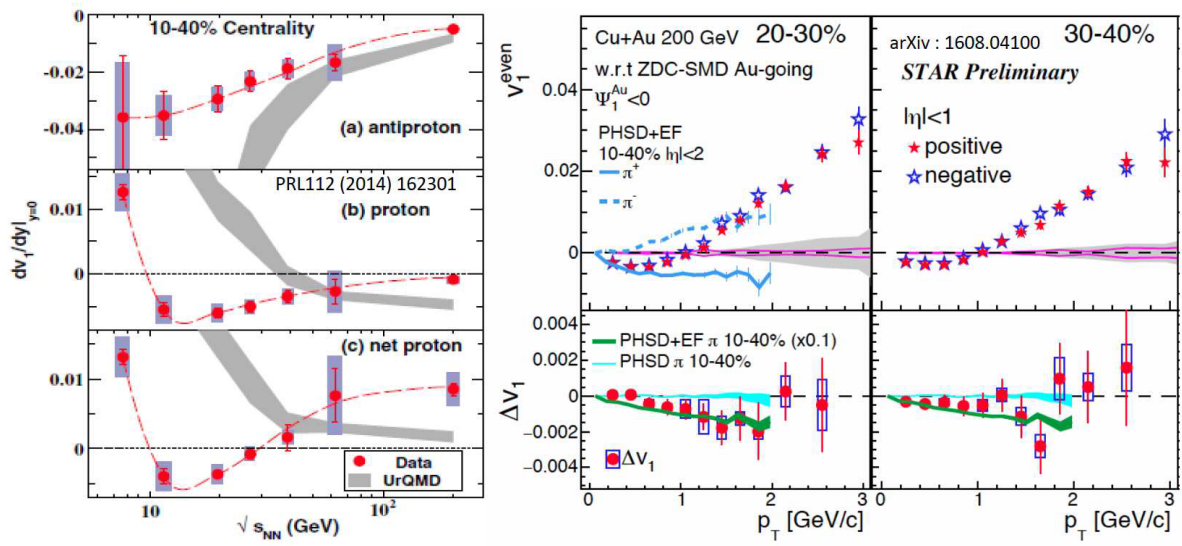

Figure 2. Directed flow $v_{1}$ slope vs $\eta$ for anti-proton, proton and net-proton as a function of colliding beam energy in $\mathrm{Au}+\mathrm{Au}$ collisions (left) [3]. Charge dependence of directed flow $v_{1}$ vs $p_{T}$ in asymmetric $\mathrm{Cu}+\mathrm{Au}$ collisions (right) [4].

reaction plane) are shown in Fig.3 (left) [5] and $v_{2}$ differences between negative and positive charged pion are shown in Fig.3 (right) as a function of charge asymmetry $A_{c h}$ of the event [6], where these observations are consistent with the expectations from the chiral magnetic effects and waves $[5,6]$.

\section{Elliptic, higher order harmonic flow and correlations}

Triggered by the ridge observation in high-multiplicity $\mathrm{p}+\mathrm{p}$ collisions at LHC energies, flow and correlation studies in small systems; $\mathrm{p}+\mathrm{A}, \mathrm{d}+\mathrm{A}$ and ${ }^{3} \mathrm{He}+\mathrm{A}$ collisions are carried out to investigate the collective phenomena in small systems at RHIC energies. Fig.4 shows the elliptic and triangular event anisotropy measurements in high multiplicity (central) events in these small collision systems, 

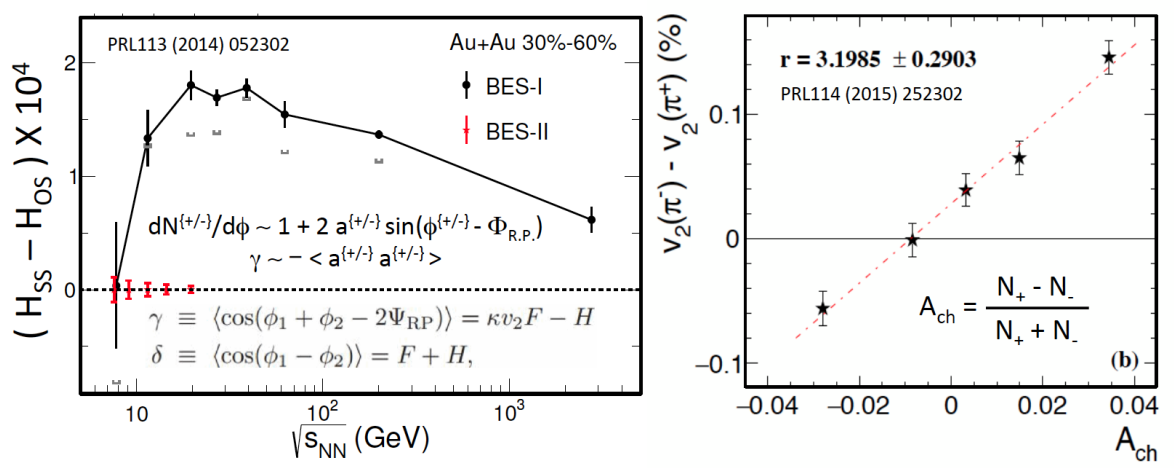

Figure 3. Possible signature of chiral magnetic effects and waves observed as charge asymmetry in 3-particle correlation (left) [5] and as charge dependent $v_{2}$ (right) [6].



Figure 4. Event anisotropy $v_{2}$ and $v_{3}$ measurements in small collision systems, $\mathrm{p}+\mathrm{Au}(\mathrm{left}), \mathrm{d}+\mathrm{Au}$ (middle) and ${ }^{3} \mathrm{He}+\mathrm{Au}$ (right) collisions at $200 \mathrm{GeV}[7-9]$.

where results are remarkably well reproduced by hydrodynamical models and transport simulations [7-9].

The higher order event anisotropy measurements for identified particles are shown in Fig.5 (left), where the hydrodynamics-like mass dependence at low $P_{T}$ region and quark coalescence-like constituent quark number dependence at intermediate $P_{T}$ region are confirmed also for the higher event anisotropy [10]. Triangular event anisotropy $v_{3}$ normalized by the system energy density; $(\mathrm{dN} / \mathrm{d} \eta) /\left(\mathrm{N}_{\text {part }} / 2\right)$ are shown as a function of colliding beam energy in Fig.5 (right), where nonmonotonic variation is observed around $\sqrt{\mathrm{S}_{\mathrm{NN}}}=10-30 \mathrm{GeV}$ [11], where this non-monotonic behavior seems to be occurred at similar beam energy region as $v_{1}$ slope in Fig.2 and as charge asymmetry from B-field in Fig.3.

Higher order event plane angles are utilized to study HBT quantum interferometry correlation in order to measure the geometrical size, duration time as well as the shape of the emission source of the colliding zone. The extracted sideward $R_{S}$ and outward $R_{o}$ radius parameters are shown in Fig.6 

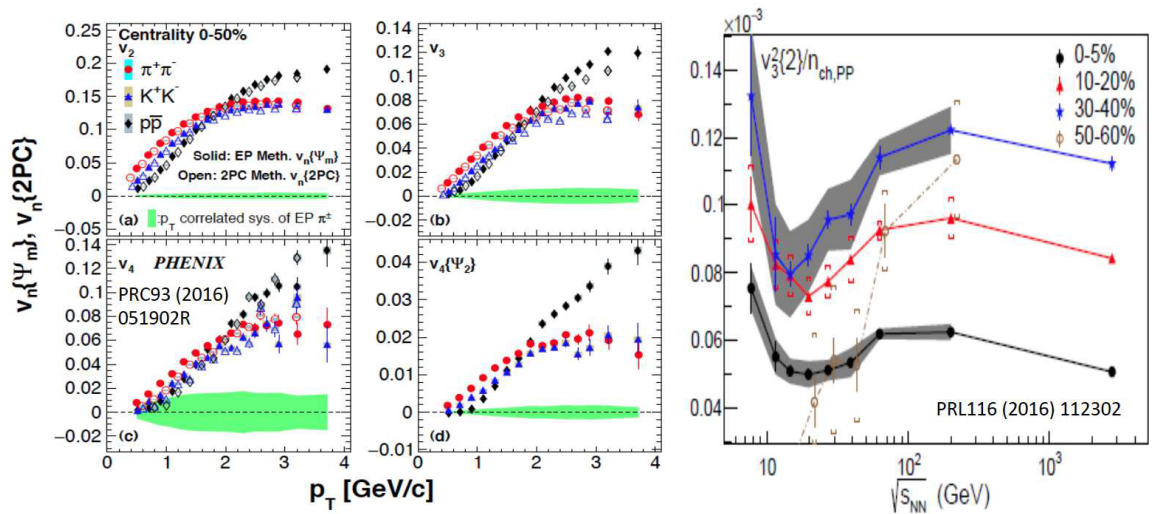

Figure 5. Higher order event anisotropy measurements $v_{2}\left(\Psi_{2}\right), v_{3}\left(\Psi_{3}\right), v_{4}\left(\Psi_{4}\right)$ and $v_{4}\left(\Psi_{2}\right)$ for identified particles $\pi^{+,-}, K^{+,-}, p$ and $\bar{p}$ (left) [10]. Beam energy dependence of $v_{3}$ normalized by the system energy density (right) [11].
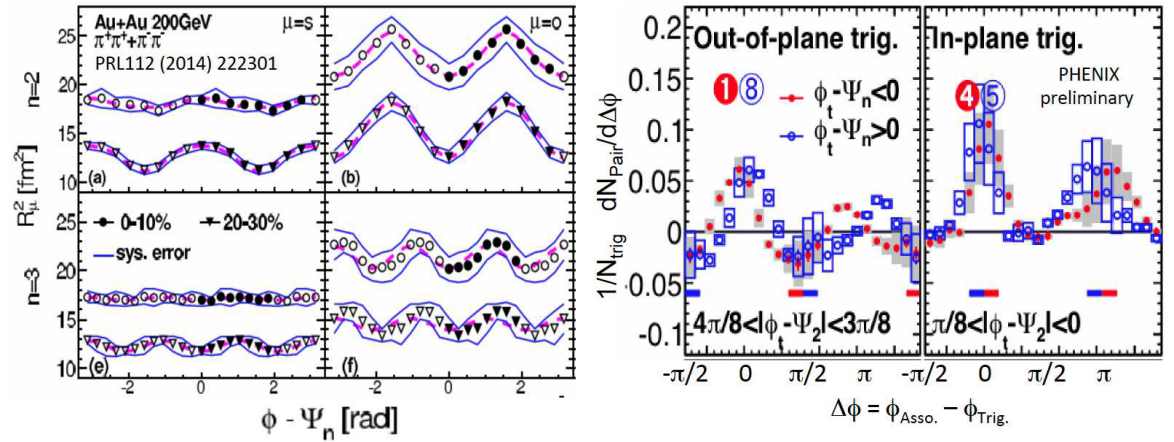

Figure 6. Azimuthal angle dependence of extracted HBT radius parameters $R_{s}^{2}$ and $R_{o}^{2}$ with respect to the 2nd and 3rd order event planes (left) [12]. Trigger angle dependence of di-hadron azimuthal correlation with respect to the 2nd order event plane (right) [13].

(left) as a function of relative pair angle from the 2 nd and the 3 rd order event planes, the measured eccentricity $\epsilon_{2, \text { final }}$ at the end of freeze-out is found to be smaller (closer to the isotropic distribution)

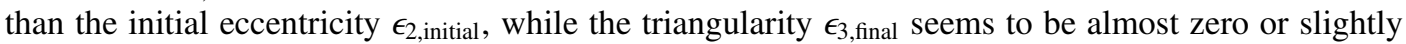
reversed at the end of freeze-out, because of the very strong radial expansion [12]. Di-hadron correlation yields per trigger are shown in Fig.6 (right) by selecting trigger particle at in-plane or out-of-plane directions including left/right dependent trigger selections with respect to the 2nd order event plane, where the correlation yields and shapes are clearly different for various trigger angle cases, which could be caused by path-length dependence of jet modification as well as flow-jet interplay coming from jet-medium interaction inside the expanding medium [13]. 



Figure 7. Heavy flavor electron suppression $R_{A A}$ and elliptic event anisotropy $v_{2}$ at RHIC (left) [14]. Charm and bottom separated electron $R_{A A}$ (top-right) [15] and reconstructed $D^{0}$ meson $v_{2}$ compared with strangeness hadrons (bottom-right) [16].

\section{Heavy quarks, photon flow and fluctuations}

Heavy quark flow and suppression have been observed to be rather significant at RHIC energies, which leads to the conclusion of strongly-interacting Quark Gluon Plasma (sQGP) formation at RHIC as shown in Fig.7 (left) [14]. Recent improved measurements especially in terms of charm and bottom quark separation are seen in Fig.7 (right panels) using new vertex detectors in both PHENIX experiment with Silicon Vertex Detector (VTX) and STAR experiment with Heavy Flavor Tracker (HFT), where the charm and bottom separated heavy flavor electron $R_{A A}$ is shown in top-right panel of Fig.7 and the identified $D^{0}$ meson $v_{2}$ is shown in bottom-right panel of Fig.7. Possible hits of heavy quark mass dependence of flow and suppression could be seen $[15,16]$.

Direct photon production at RHIC energy have been confirmed to be as a penetrating probe $\left(R_{A A} \sim 1\right.$ and $\left.v_{2} \sim 0\right)$ at high $p_{T}$ region above 5-6 GeV/c [17], while the enhanced photon production at lower $p_{T}$ region are considered as thermal photon radiation from early stage of hot matter [18] as shown in Fig.8 (left). Therefore, it has been very much surprising to observe quite large magnitude of elliptic and triangular event anisotropies $v_{2}$ and $v_{3}$ at low $p_{T}$ region below $4 \mathrm{GeV} / \mathrm{c}$ as shown in Fig.8 (middle- top and bottom panels), which is somewhat contradicting to have such large $v_{2,3}$ for early photons emitted before the strong expansion, where this needs further investigations [19]. Fluctuation of conserved quantity is expected to be sensitive to the critical point as an end point of 1st order phase transition, because a sudden change of correlation length is expected close to the critical point. Higher order cumulant ratio of net-proton distribution (ratio of 4th-order over 2nd-order cumulant) is shown as a function of beam energy [20], where there might be some possible critical fluctuations at similar beam energy region again around $10-30 \mathrm{GeV}$, although the statistical and systematic errors of the measurements are comparable with the magnitude of the observed possible critical fluctuation. This is the main reason why the beam energy scan phase 2 program will be pursued in the next 3-5 years at RHIC. 

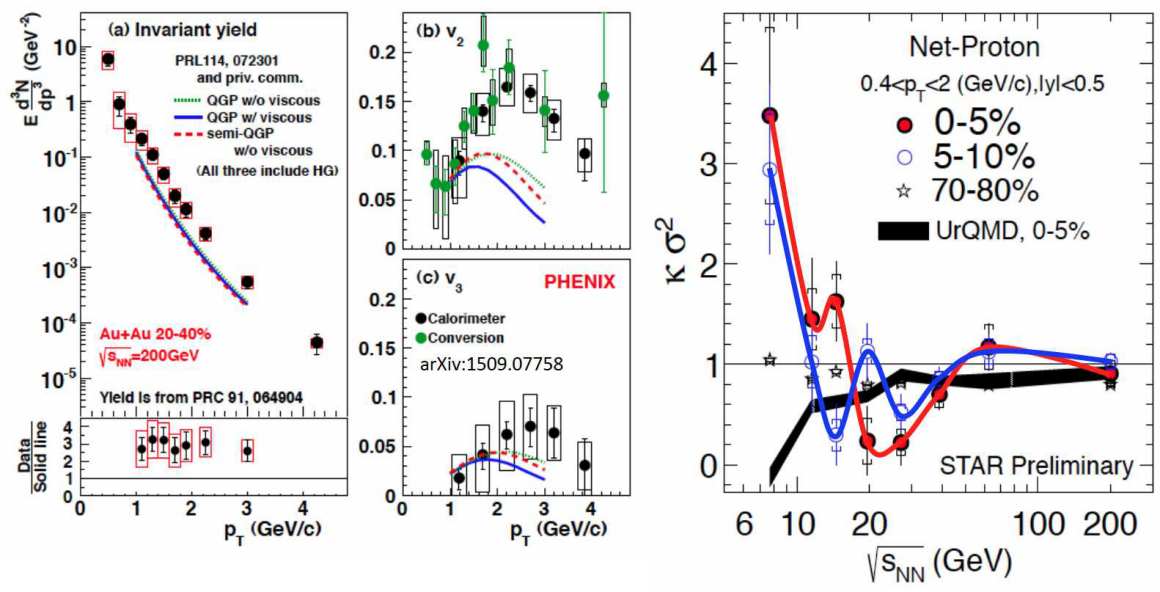

Figure 8. Low $p_{T}$ direct photon yield and azimuthal anisotropy $v_{2,3}$ (left) [19]. Cumulant ratio of 4th-order over 2nd-order $\left(\kappa \sigma^{2}\right)$ of net-proton distribution as a function of beam energy (right) [20].

\section{References}

[1] S. Chatterjee et al., Adv. High Energy Phys. 2015, 349013 (2015);

V. Bairathi, QM2015; B. Mohanty, SQM2016

[2] B. I. Abelev et al. (STAR Collaboration), Phys. Rev. C79, 034909 (2009);

B. Abelev et al. (ALICE Collaboration), Phys. Rev. C88, 044910 (2013)

[3] L. Adamczyk et al. (STAR Collaboration), Phys. Rev. Lett. 112, 162301 (2014)

[4] L. Adamczyk et al. (STAR Collaboration), arXiv:1608.04100

[5] L. Adamczyk et al. (STAR Collaboration), Phys. Rev. Lett. 113, 052302 (2014)

[6] L. Adamczyk et al. (STAR Collaboration), Phys. Rev. Lett. 114, 252302 (2015)

[7] C. Aidala et al. (PHENIX Collaboration), arXiv:1609.02894

[8] A. Adare et al. (PHENIX Collaboration), Phys. Rev. Lett. 114, 192301 (2015)

[9] A. Adare et al. (PHENIX Collaboration), Phys. Rev. Lett. 115, 142301 (2015)

[10] A. Adare et al. (PHENIX Collaboration), Phys. Rev. C93, 051902R (2016)

[11] L. Adamczyk et al. (STAR Collaboration), Phys. Rev. Lett. 116, 112302 (2016)

[12] A. Adare et al. (PHENIX Collaboration), Phys. Rev. Lett. 112, 222301 (2014)

[13] T. Todoroki for the PHENIX Collaboration, Nucl. Phys. A904-905, 495c (2013)

[14] A. Adare et al. (PHENIX Collaboration), Phys. Rev. Lett. 98, 172301 (2007)

[15] A. Adare et al. (PHENIX Collaboration), Phys. Rev. C93, 034904 (2016)

[16] L. Adamczyk et al. (STAR Collaboration), Phys. Rev. Lett. 116, 062301 (2016)

[17] S. Afanasiev et al. (PHENIX Collaboration), Phys. Rev. Lett. 109, 152302 (2012);

A. Adare et al. (PHENIX Collaboration), Phys. Rev. Lett. 109, 122302 (2012)

[18] A. Adare et al. (PHENIX Collaboration), Phys. Rev. C91, 064904 (2015)

[19] A. Adare et al. (PHENIX Collaboration), arXiv:1509.07758

[20] X. Luo for the STAR Collaboration), QM2015 